\title{
Synthesis of thiophene-pyrazole conjugates as potent antimicrobial and radical scavengers
}

\author{
Malledevarapura Gurumurthy Prabhudeva ${ }^{a}$, Nagamallu Renukab ${ }^{b}$ and Kariyappa Ajay Kumar ${ }^{a^{*}}$
}

${ }^{a}$ Department of Chemistry, Yuvaraja College, University of Mysore, Mysuru-570005, India

${ }^{b}$ Department of Chemistry, GSSS Institute of Engineering and Technology For Women, Mysuru 570 016, India

\begin{tabular}{l}
\hline C H R O N I C L E \\
\hline Article history: \\
Received April 28, 2018 \\
Received in revised form \\
June 29, 2018 \\
Accepted August 2, 2018 \\
Available online \\
August 2, 2018 \\
\hline Keywords: \\
Antimicrobial \\
Antioxidant \\
Chalcone \\
Cyclocondensation \\
Radical scavengers
\end{tabular}
\begin{abstract}
A B S T R A C T
The current study presents the synthesis of thiophene-appended pyrazoles through $3+2$ annulations of chalcones $3(\mathbf{a}-\mathbf{g})$ with aryl hydrazine hydrochlorides $\mathbf{4}(\mathbf{a}-\mathbf{d})$ in acetic acid (30\%) under reflux conditions produced the thiophene-pyrazole hybrids $\mathbf{5}(\mathbf{a}-\mathbf{g})$ in good yields. Structures of synthesized new pyrazoles were confirmed by spectral studies, and elemental analysis. Further, preliminary biological evaluation studies show that compounds $\mathbf{5 b}$ and $\mathbf{5 f}$ having chloro substitution only in the thiophene ring exhibited excellent inhibition (12.5-25.0 $\mu \mathrm{g} / \mathrm{mL}$ ) against all the tested organisms in comparison with that of the standard. Compounds, $\mathbf{5 a}, \mathbf{5 c}$ and $\mathbf{5 g}$ having electronegative chloro substitutions each in the aromatic and thiophene rings showed excellent (12.423-31.213 $\left.\mu \mathrm{g} \mathrm{mL}^{-1}\right)$ DPPH radical scavenging potencies. The synthesis of pyrazoline derivatives and the efficacy of some of the synthesized molecules as antimicrobial and antioxidant agents validate the significance of this study.
\end{abstract}

(C) 2018 Growing Science Ltd. All rights reserved.

\section{Introduction}

An interest in discovery, design and synthesis of novel small-molecules with antimicrobial and radical scavenging effects is propelling research in the wider research community in order to prevent the deleterious effects that free-oxide radicals can inflict upon the human body. Duloxetine is a "blockbuster" antidepressant without any adverse effect associated with the formation of RMs due to the judicious conjugation of thiophene moiety with naphthalene, ${ }^{1}$ which facilitates the potentiality of employing this functional group for the synthesis of small-molecules with desired biological effect. Chalcones are the principal precursors for the synthesis of bioactive small molecules such as benzothiazepines, ${ }^{2}$ pyrazolines, ${ }^{3}$ isoxazolines, ${ }^{4}$ cylopropanes, ${ }^{5}$ oxadiazoles, ${ }^{6}$ etc., The chalcones are most commonly synthesized via Claisen-Schmidt reaction of an aromatic aldehyde with acetophenones. ${ }^{7}$ Chalcones has gained importance due to their simple structures and diverse pharmacological applications. ${ }^{8}$

Design and synthesis of simple heterocycles with various bioactivities is a worthwhile contribution in organic synthesis. The compounds with pyrazole core are the most important class in active

* Corresponding author. 
pharmaceutical drugs and remain the choice for anti-inflammatory agents in spite of multiple attempts at exploring alternative scaffolds. ${ }^{9,10}$ Amongst the various methods available in the literature for the synthesis of pyrazole scaffolds, most commonly employed being; a base catalyzed reaction of hydrazines with 1,3-dicarbonyl compounds, ${ }^{11}$ 1,3-dipolar cycloaddition of hydrazones to alkenes, ${ }^{12}$ and via Vilsmeier-Haack reaction of arylhydrazones. ${ }^{13}$ Further, it is emphasized here that pyrazoles are regarded as promising molecules with potential applications in bioorganic chemistry. Pyrazoles were known to exhibit anticancer, ${ }^{14}$ antimicrobial, ${ }^{15}$ anesthetic, ${ }^{16}$ antioxidant, ${ }^{17}$ and analgesic ${ }^{18}$ activities. In view of the wide range of synthetic and biological applications of pyrazoles, we herein report the synthesis of derivatives of pyrazoles and the results of their in vitro evaluation for antimicrobial and DPPH radical scavenging activities. The demonstrated synthesis paves the way for future efforts at synthesizing pyrazoles that could find widespread applications in medicinal chemistry.

\section{Results and Discussion}

\subsection{Chemistry}

Initially, the intermediate 3-aryl-1-(5-chlorothiophen-2-yl)prop-2-en-1-ones, 3(a-d), were synthesized by base catalyzed reaction of 2-acetyl-5-chlorothiophene, $\mathbf{1}$, with aromatic aldehydes, $\mathbf{2}(\mathbf{a}-$ d) in methyl alcohol. Then, the reaction of $\mathbf{3}(\mathbf{a}-\mathbf{d})$ and arylhydrazine hydrochloride $\mathbf{4}(\mathbf{a}-\mathbf{b})$ in aqueous acetic acid under reflux conditions produced pyrazole derivatives $\mathbf{5}(\mathbf{a}-\mathbf{g})$ (Fig. 1). ${ }^{1} \mathrm{H} \mathrm{NMR},{ }^{13} \mathrm{C}$ NMR, MS and elemental analysis provided the structural proof for the compounds, $\mathbf{3}(\mathbf{a}-\mathbf{d})$ and $\mathbf{5}(\mathbf{a}-\mathbf{g})$.
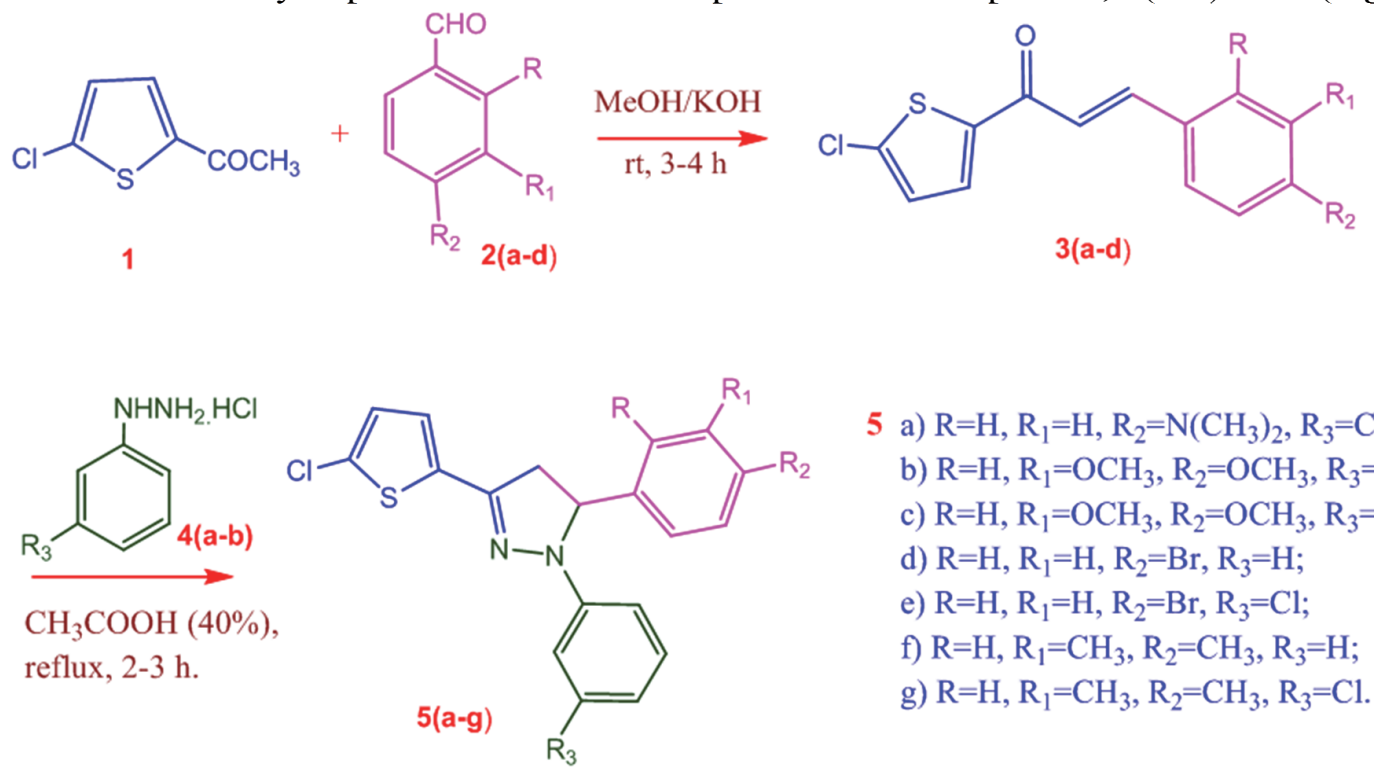
5 a) $\mathrm{R}=\mathrm{H}, \mathrm{R}_{1}=\mathrm{H}, \mathrm{R}_{2}=\mathrm{N}\left(\mathrm{CH}_{3}\right)_{2}, \mathrm{R}_{3}=\mathrm{Cl}$;
b) $\mathrm{R}=\mathrm{H}, \mathrm{R}_{1}=\mathrm{OCH}_{3}, \mathrm{R}_{2}=\mathrm{OCH}_{3}, \mathrm{R}_{3}=\mathrm{H}$;
c) $\mathrm{R}=\mathrm{H}, \mathrm{R}_{1}=\mathrm{OCH}_{3}, \mathrm{R}_{2}=\mathrm{OCH}_{3}, \mathrm{R}_{3}=\mathrm{Cl}$;
d) $\mathrm{R}=\mathrm{H}, \mathrm{R}_{1}=\mathrm{H}, \mathrm{R}_{2}=\mathrm{Br}, \mathrm{R}_{3}=\mathrm{H}$;
e) $\mathrm{R}=\mathrm{H}, \mathrm{R}_{1}=\mathrm{H}, \mathrm{R}_{2}=\mathrm{Br}, \mathrm{R}_{3}=\mathrm{Cl}$;
f) $\mathrm{R}=\mathrm{H}, \mathrm{R}_{1}=\mathrm{CH}_{3}, \mathrm{R}_{2}=\mathrm{CH}_{3}, \mathrm{R}_{3}=\mathrm{H}$;
g) $\mathrm{R}=\mathrm{H}, \mathrm{R}_{1}=\mathrm{CH}_{3}, \mathrm{R}_{2}=\mathrm{CH}_{3}, \mathrm{R}_{3}=\mathrm{Cl}$.

Fig. 1. Schematic diagram for the synthesis of pyrazoles, $\mathbf{5}(\mathbf{a}-\mathbf{g})$

The reaction of 2-acetyl-5-chlorothiophene 1, and aromatic aldehydes, $\mathbf{2}(\mathbf{a}-\mathbf{d})$, in the presence of potassium hydroxide produced 3-aryl-1-(5-chlorothiophen-2-yl) prop-2-en-1-ones, 3(a-d), in moderate yields. In ${ }^{1} \mathrm{H}$ NMR spectra, compounds 3(a-d) showed a doublets for one proton at $\delta$ 7.103$7.110 \mathrm{ppm}(\mathrm{J}=16.2 \mathrm{MHz})$ for $\mathrm{CH}=$ proton; and at $\delta 8.020-8.130 \mathrm{ppm}(\mathrm{J}=16.1 \mathrm{MHz})$ for $=\mathrm{CH}$ protons of the double bond. The coupling constant values of these doublets ranging from $J=16.1-16.2 \mathrm{~Hz}$, indicating the $(E)$-configuration around the $\mathrm{C}=\mathrm{C}$ bond. The signals due to methoxy protons appear as singlet at $\delta 3.854 \mathrm{ppm}$; and methyl protons appear at $\delta 2.324 \mathrm{ppm}$. Further, all compounds showed an array of signals appeared in the aromatic region were unambiguously assigned to thiophene and aromatic protons. In the ${ }^{13} \mathrm{C}$ NMR spectra, all synthesised compounds $\mathbf{3}(\mathbf{a}-\mathbf{d})$ showed signals due to methoxy carbons at $\delta 56.20 \mathrm{ppm}$; methyl carbons at $\delta 20.42 \mathrm{ppm} ; \mathrm{CH}=$ carbons at $\delta 120.10-121.20$ 
ppm; $=\mathrm{CH}$ carbons at $\delta 144.86-146.22 \mathrm{ppm}$ and carbonyl $(\mathrm{C}=\mathrm{O})$ carbons at $\delta 183.40-182.56 \mathrm{ppm}$. The signals observed in the aromatic carbons region were due to thiophene and aromatic ring carbons.

In search of new potent antimicrobial and radical scavenging agents, we were successful in synthesising a series of new thiophene-pyrazole hybrids $\mathbf{5}(\mathbf{a}-\mathbf{g})$ by the acid catalyzed reaction of chalcones 3(a-d) with arylhydrazine hydrochlorides $4(\mathbf{a}-\mathbf{b})$ in good yields. ${ }^{1} \mathrm{H}$ NMR spectra of compounds $\mathbf{5}(\mathbf{a}-\mathbf{g})$ showed that, the methylene protons of C-4 atom of newly formed pyrazole ring exhibited typical ABX spin and are of diastereotopic nature. For instance, in their spectra, the $\mathrm{C}_{4}-\mathrm{H}_{\mathrm{a}}$ proton appears as doublet of doublet at $\delta 3.113-3.128(\mathrm{dd}, 1 \mathrm{H}, J=6.1-7.2 \mathrm{~Hz}$ and $J=16.0-16.8 \mathrm{~Hz}$ ) ppm; whereas, $\mathrm{C}_{4}-\mathrm{H}_{\mathrm{b}}$ proton appears as doublet of doublet at $\delta 3.740-3.780(\mathrm{dd}, J=12.0-12.6 \mathrm{~Hz}$ and $J=7.0$ $7.5 \mathrm{~Hz}) \mathrm{ppm}$, respectively. Instead of appearing as a triplet, $\mathrm{C}_{5}-\mathrm{H}$ resonates with both $\mathrm{C}_{4}-\mathrm{H}_{\mathrm{a}}$ and $\mathrm{C}_{4}-\mathrm{H}_{\mathrm{b}}$ and appears as doublet of doublet at $\delta 5.238-5.251$ (dd, $J=6.0-6.4 \mathrm{~Hz}$ and $J=12.0-12.4 \mathrm{~Hz} \mathrm{ppm}$. The signals appeared as singlets due to aromatic methyl protons in the region $\delta 2.295-2.230 \mathrm{ppm}$; methoxy protons at $\delta 3.845-3.850 \mathrm{ppm}$; and $N$-methyl protons at $\delta 3.030 \mathrm{ppm}$. Further, all compounds showed an array of signals as doublet and multiplet in the aromatic proton absorption range and were unambiguously assigned to thiophene and aromatic ring protons.

In the ${ }^{13} \mathrm{C}$ NMR spectra, compounds $\mathbf{5}(\mathbf{a}-\mathbf{g})$ showed the signals due to the C-4, C-5 and C-3 carbons of newly formed pyrazole ring correspondingly at $\delta 42.54-44.25,63.10-63.90$ and $147.30-149.64 \mathrm{ppm}$. The appearance of signals for C-4 at $\delta 42.54-44.25 \mathrm{ppm}$ and C-5 at $\delta 63.10-63.90 \mathrm{ppm}$ confirms that the ring is of partially reduced dihydropyrazole form. The signals due to substitution carbons such as methyl carbons in the region $\delta 19.80-20.61 \mathrm{ppm}$; methoxy carbons at $\delta 55.45-55.48 \mathrm{ppm}$; and $N$-methyl carbons at $\delta 40.36 \mathrm{ppm}$. Further, all compounds showed an array of signals in the aromatic region and were unambiguously assigned to thiophene and aromatic ring carbons.

All designed series of compounds, 3(a-d) and 5(a-g) showed a base peak corresponding to their molecular masses and also ${ }^{37} \mathrm{Cl},{ }^{81} \mathrm{Br}$ isotope peaks. Further, all compounds showed satisfactory elemental analyses compared with theoretical values, which strongly favour the formation of the designed products.

\subsection{Biological evaluations}

\subsubsection{Antimicrobial activity}

The new synthesized pyrazole derivatives $\mathbf{5}(\mathbf{a - g})$ were screened for their antibacterial and antifungal activity by serial dilution method. ${ }^{19}$ The experiments were carried out in triplicate; the results were taken as a mean of three determinations $(n=3)$. For antibacterial studies, the bacteria species Staphylococcus aureus, Escherichia coli and Pseudomonas aeruginosa; for antifungal studies, Aspergillus niger, Aspergillus flavus and Candila albicans were used as microbial strains. The antibiotics ciprofloxacin and nystatin were used as reference drugs against bacteria and fungi species respectively. The results of MIC's of the synthesized compounds against bacteria and fungi species were summarized in Table 1 respectively.

Preliminary studies reveal that the synthesized series of new pyrazole derivatives $\mathbf{5}(\mathbf{a}-\mathbf{g})$ showed broad spectrum of antimicrobial activities against the tested species. Amongst the series, compounds $\mathbf{5 b}$ and $\mathbf{5 f}$ having chloro substitution only in the thiophene ring exhibited excellent inhibition (12.5$25.0 \mu \mathrm{g} / \mathrm{mL}$ ) against all the tested organisms in comparison with that of the standard. Promising inhibition was shown by compound $\mathbf{5 a}$ against $C$. albicans $(25.0 \mu \mathrm{g} / \mathrm{mL})$, and $\mathbf{5 c}$ against $P$. aeruginosa $(12.5 \mu \mathrm{g} / \mathrm{mL})$. Compounds $\mathbf{5 d}$ and $\mathbf{5 e}$ having bromo substitutions in the aromatic ring showed poorer inhibition (50.0-100.0 $\mu \mathrm{g} / \mathrm{mL})$ against the tested species. Compound $\mathbf{5 c}$ showed moderate inhibition 
against $S$. aureus $(75.0 \mu \mathrm{g} / \mathrm{mL})$, A. niger $(100.0 \mu \mathrm{g} / \mathrm{mL})$, and A. flavus $(100.0 \mu \mathrm{g} / \mathrm{mL})$; and $\mathbf{5 c}$ against A. flavus $(100.0 \mu \mathrm{g} / \mathrm{mL})$, and C. albicans $(75.0 \mu \mathrm{g} / \mathrm{mL})$, and $\mathbf{5 c}$ against $S$. aureus $(75 \mu \mathrm{g} / \mathrm{mL})$ and $E$. coli $(75.0 \mu \mathrm{g} / \mathrm{mL})$ species. Moderate inhibition showed by compounds, $\mathbf{5 a}$ against E. coli $(37.5 \mu \mathrm{g} / \mathrm{mL})$ and $P$. aeruginosa $(25.0 \mu \mathrm{g} / \mathrm{mL}) ; \mathbf{5 c}$ against $S$. aureus $(37.5 \mu \mathrm{g} / \mathrm{mL})$, E. coli $(37.5 \mu \mathrm{g} / \mathrm{mL})$, and A. niger $(75.0 \mu \mathrm{g} / \mathrm{mL})$; and $5 \mathrm{~g}$ against $P$. aeruginosa $(25.0 \mu \mathrm{g} / \mathrm{mL})$, and $A$. niger $(100.0 \mu \mathrm{g} / \mathrm{mL})$ organisms.

Table 1. Minimum inhibitory concentrations (MIC's) in $\mu \mathrm{g} / \mathrm{mL}^{*}$ of compounds $\mathbf{5}(\mathbf{a}-\mathbf{g})$ against bacteria and fungi species

\begin{tabular}{|c|c|c|c|c|c|c|}
\hline Entry & S. aureus & E. coli & P. aeruginosa & A. niger & A. flavus & C. albicans \\
\hline $5 a$ & 75.0 & 37.5 & 25.0 & 100.0 & 100.0 & 25.0 \\
\hline $5 b$ & 25.0 & 25.0 & 12.5 & 25.0 & 25.0 & 25.0 \\
\hline $5 c$ & 37.5 & 37.5 & 12.5 & 75.0 & 100.0 & 75.0 \\
\hline $5 d$ & 75.0 & 100.0 & 50.0 & 100.0 & 100.0 & 75.0 \\
\hline $5 e$ & 100.0 & 75.0 & 75.0 & 100.0 & 100.0 & 100.0 \\
\hline $5 f$ & 25.0 & 25.0 & 12.5 & 25.0 & 25.0 & 25.0 \\
\hline $5 \mathrm{~g}$ & 75.0 & 75.0 & 25.0 & 100.0 & 100.0 & 75.0 \\
\hline Ciprofloxacin & $\begin{array}{c}25.0 \\
25.0^{20}\end{array}$ & $\begin{array}{c}25.0 \\
25.0^{20}\end{array}$ & $\begin{array}{c}12.5 \\
12.5^{19}\end{array}$ & -- & -- & -- \\
\hline Nystatin & -- & -- & -- & $\begin{array}{c}50.0 \\
50.0^{20}\end{array}$ & $\begin{array}{c}50.0 \\
50.0^{20}\end{array}$ & $\begin{array}{c}25.0 \\
25.0^{19}\end{array}$ \\
\hline
\end{tabular}

*Results are expressed as mean of three determinations $(\mathrm{n}=3)$

\subsubsection{DPPH radical scavenging activity}

The DPPH radical scavenging ability of the synthesized compounds $\mathbf{5}(\mathbf{a}-\mathbf{g})$ was performed by a reported procedure..$^{21}$ The experiments were performed with different aliquots of test samples $(25,50$, 75 and $100 \mu \mathrm{g} \mathrm{mL}^{-1}$ ) in methanol and the absorbance was read against blank at $517 \mathrm{~nm}$ in an ELICO SL $159 \mathrm{UV}$ visible spectrophotometer. Tests were carried out in triplicate and the results are expressed as $\mathrm{I} \% \pm$ standard deviations and were summarized in Table 2.

Preliminary studies of synthesized pyrazoline derivatives moderate to good DPPH radical scavenging abilities because of their H-donating capacity. Results of the investigations shows that the compounds $\mathbf{5 d}$ and $\mathbf{5 e}$ were having bromo substitutions in the aromatic rings showed moderate (28.500$\left.55.900 \mu \mathrm{g} \mathrm{mL}^{-1}\right)$. Compounds, 5a, 5c and $\mathbf{5 g}$ having electronegative chloro substitutions each in the aromatic and thiophene rings showed excellent (12.423-31.213 $\left.\mu \mathrm{g} \mathrm{mL}^{-1}\right)$ radical scavenging potencies. Compounds $\mathbf{5 b}$ and $\mathbf{5 f}$ have showed moderate activities $\left(12.423-31.213 \mu \mathrm{g} \mathrm{mL}^{-1}\right)$ in comparison with the standard ascorbic acid.

Table 2. DPPH Radical Scavenging ability (in \%)* of compounds $\mathbf{5}(\mathbf{a - g})$ at different concentrations

\begin{tabular}{cllll}
\hline Entry & $25\left(\mu \mathrm{g} \mathrm{mL}^{-1}\right)$ & $\left.50(\mu \mathrm{g} \mathrm{mL})^{-1}\right)$ & $75\left(\mu \mathrm{g} \mathrm{mL}^{-1}\right)$ & $100\left(\mu \mathrm{g} \mathrm{mL} \mathrm{mL}^{-1}\right)$ \\
\hline 5a & $14.200 \pm 0.54$ & $21.125 \pm 0.47$ & $26.140 \pm 0.50$ & $31.121 \pm 0.48$ \\
5b & $18.220 \pm 0.50$ & $24.330 \pm 0.53$ & $32.212 \pm 0.47$ & $41.200 \pm 0.51$ \\
5c & $12.423 \pm 0.34$ & $19.100 \pm 0.35$ & $23.140 \pm 0.50$ & $29.011 \pm 0.43$ \\
5d & $28.500 \pm 0.45$ & $37.104 \pm 0.44$ & $42.330 \pm 0.45$ & $54.006 \pm 0.57$ \\
5e & $29.808 \pm 0.50$ & $38.543 \pm 0.60$ & $43.755 \pm 0.65$ & $55.900 \pm 0.49$ \\
5f & $19.110 \pm 0.66$ & $25.410 \pm 0.45$ & $30.527 \pm 0.32$ & $44.440 \pm 0.42$ \\
5g & $15.000 \pm 0.58$ & $20.150 \pm 0.68$ & $25.700 \pm 0.54$ & $31.213 \pm 0.55$ \\
Ascorbic acid & $11.194 \pm 0.29$ & $16.186 \pm 0.51$ & $22.904 \pm 0.56$ & $26.655 \pm 0.62$ \\
& $15.080 \pm 0.89^{22}$ & $17.870 \pm 0.89^{22}$ & $21.980 \pm 0.31^{22}$ & $24.250 \pm 0.22^{22}$ \\
\hline
\end{tabular}

*Results are expressed as mean of three determinations $(n=3) \pm$ Standard Deviation (SD)

\section{Conclusions}

The synthesis of pyrazoline derivatives and the efficacy of some of the synthesized molecules as antimicrobial and antioxidant agents validate the significance of this study. Preliminary studies show 
that compounds $\mathbf{5 b}$ and $\mathbf{5 f}$ having chloro substitution only in the thiophene ring exhibited significant excellent inhibition $(12.5-25.0 \mu \mathrm{g} / \mathrm{mL})$ against all the tested organisms in comparison with that of the standard. Compounds, 5a, 5c and $\mathbf{5 g}$ having electronegative chloro substitutions each in the aromatic and thiophene rings showed excellent (12.423-31.213 $\left.\mu \mathrm{g} \mathrm{mL}^{-1}\right)$ DPPH radical scavenging potencies.

\section{Acknowledgements}

The authors are grateful to IOE Instrumentation facility, University of Mysore, for recording spectra of the compounds reported.

\section{Experimental}

\subsection{Materials and Methods}

Melting points were determined by an open capillary tube method and are uncorrected. ${ }^{1} \mathrm{H}$ NMR and ${ }^{13} \mathrm{C}$ NMR spectra were recorded on Agilent-NMR $400 \mathrm{MHz}$ and $125 \mathrm{MHz}$ spectrometer respectively. The chemical shifts are expressed in $\delta \mathrm{ppm}$. Mass spectra were obtained on GC-EI-MS Agilent 7890A model spectrometer. Elemental analysis was obtained on a Thermo Finnigan Flash EA $1112 \mathrm{CHN}$ analyzer.

4.2. General procedure for synthesis of chalcones, 3(a-d): To a solution mixture of 5-chloro-2acetylthiophene, $1(10 \mathrm{mmol})$ and aromatic aldehydes, $\mathbf{2}(\mathbf{a}-\mathbf{d})(10 \mathrm{mmol})$ in methyl alcohol, potassium hydroxide solution $(40 \%, 2 \mathrm{~mL})$ was added. Then the solution mixture was stirred at room temperature for 3-4 h. The progress of the reaction was monitored by TLC. After the completion, the reaction mixture was cooled to room temperature and poured into ice cold water. Solids separated were filtered, washed successively with cold hydrochloric acid (5\%) and cold water. Crude solids were recrystallized from methyl alcohol to obtain the compounds $\mathbf{3}(\mathbf{a}-\mathbf{d})$.

4.3. General procedure for synthesis of pyrazoles, $\mathbf{5}(\mathbf{a}-\mathbf{g})$ : A solution mixture of chalcones, $\mathbf{3}(\mathbf{a}-\mathbf{d})$ (10 $\mathrm{mmol})$ and phenylhydrazine hydrochlorides, $4(\mathbf{a}-\mathbf{b})(10 \mathrm{mmol})$ in aqueous acetic acid (30\%) was refluxed for 2-3 $\mathrm{h}$. The progress of the reaction was monitored by TLC. After the completion, the mixture was cooled and poured in to a crushed ice. The separated solids were filtered and washed with water. Crude solids were recrystallized from ethyl alcohol to get target molecules $\mathbf{5}(\mathbf{a}-\mathbf{g})$.

\subsection{Physical and Spectral Data}

4.3.1 1-(5-Chlorothiophen-2-yl)-3-(4-(dimethylamino)phenyl)prop-2-en-1-one, $\quad 3 a: \quad W e \quad$ have reported the synthesis and characterization earlier. ${ }^{23}$

4.3.2 1-(5-Chlorothiophen-2-yl)-3-(3,4-dimethoxyphenyl)prop-2-en-1-one, 3b: Obtained from 2acetyl-5-chlorothiophene, $\mathbf{1}(1.69 \mathrm{~g}, 10 \mathrm{mmol})$ and 3,4-dimethoxybenzaldehyde, $\mathbf{2 b}(1.66 \mathrm{~g}, 10 \mathrm{mmol})$ in $78 \%$ yield; m.p. $122-125{ }^{\circ} \mathrm{C} .{ }^{1} \mathrm{H}$ NMR $\left(\mathrm{CDCl}_{3}, \delta \mathrm{ppm}\right): 3.854\left(\mathrm{~s}, 6 \mathrm{H}, \mathrm{OCH}_{3}\right), 6.882(\mathrm{~d}, 1 \mathrm{H}, \mathrm{Ar}-\mathrm{H})$, $7.103(\mathrm{~d}, 1 \mathrm{H}, J=16.2 \mathrm{MHz}, \mathrm{CH}=), 7.202-7.565(\mathrm{~m}, 4 \mathrm{H}, \mathrm{Ar}-\mathrm{H}), 8.124(\mathrm{~d}, 1 \mathrm{H}, J=16.1 \mathrm{MHz},=\mathrm{CH}) ;{ }^{13} \mathrm{C}$ NMR ( $\left.\mathrm{CDCl}_{3}, \delta \mathrm{ppm}\right): 56.20\left(2 \mathrm{C}, \mathrm{OCH}_{3}\right), 110.35(1 \mathrm{C}), 117.90(1 \mathrm{C}), 120.10(1 \mathrm{C}, \mathrm{CH}=), 121.84(1 \mathrm{C})$, 127.60 (1C), 129.70 (1C), 134.40 (1C), 139.35 (1C), 144.30 (1C), 146.22 (1C, =CH), $149.52(1 \mathrm{C})$, 149.60 (1C), $183.40(1 \mathrm{C}, \mathrm{C}=\mathrm{O})$. MS (EI) $m / z: 310.03$ (32), 308.01 (M+, 100); Anal. calcd. for $\mathrm{C}_{15} \mathrm{H}_{13} \mathrm{ClO}_{3} \mathrm{~S}(\%)$ : C, 58.35; H, 4.24. Found: C, 58.30; H, 4.23.

4.3.3 3-(4-Bromophenyl)-1-(5-chlorothiophen-2-yl)prop-2-en-1-one, 3c: Obtained from 2-acetyl-5chlorothiophene, $1(1.69 \mathrm{~g}, 10 \mathrm{mmol})$ and 4-bromobenzaldehyde, $\mathbf{2 c}(1.84 \mathrm{~g}, 10 \mathrm{mmol})$ in $66 \%$ yield; m.p. 116-118 ${ }^{\circ} \mathrm{C} .{ }^{1} \mathrm{H}$ NMR $\left(\mathrm{CDCl}_{3}, \delta\right.$ ppm): $6.890(\mathrm{~d}, 1 \mathrm{H}, \mathrm{Ar}-\mathrm{H}), 7.110(\mathrm{~d}, 1 \mathrm{H}, J=16.2 \mathrm{MHz}, \mathrm{CH}=)$, 7.522 (d, 1H, Ar-H), 7.590 (d, 1H, J=7.2 MHz, Ar-H), 7.762 (d, 1H, J=7.2 MHz, Ar-H), 8.130 (d, 1H, $J=16.1 \mathrm{MHz},=\mathrm{CH}) ;{ }^{13} \mathrm{C} \mathrm{NMR}\left(\mathrm{CDCl}_{3}, \delta \mathrm{ppm}\right): 121.15(1 \mathrm{C}, \mathrm{CH}=), 122.80(1 \mathrm{C}), 129.61(1 \mathrm{C}), 128.34$ (1C), 128.78 (1C), 130.26 (1C), 130.48 (1C), 134.30 (1C), 134.88 (1C), 139.97 (1C), 145.20 (1C, $=\mathrm{CH}), 146.02(1 \mathrm{C}), 182.56(1 \mathrm{C}, \mathrm{C}=\mathrm{O})$. MS (EI) $\mathrm{m} / z$ : 329.91 (31), 327.91 (98), $325.90(\mathrm{M}+, 100)$; Anal. calcd. for $\mathrm{C}_{13} \mathrm{H}_{8} \mathrm{BrClOS}(\%)$ : C, 47.66; H, 2.46. Found: C, 47.63; H, 2.45. 
4.3.4 1-(5-Chlorothiophen-2-yl)-3-(2,4-dimethylphenyl)prop-2-en-1-one, 3d: Obtained from 2acetyl-5-chlorothiophene, $1(1.69 \mathrm{~g}, 10 \mathrm{mmol})$ and 2,4-dimethylbenzaldehyde, $\mathbf{2 d}(1.34 \mathrm{~g}, 10 \mathrm{mmol})$ in 82\% yield; m.p. $110-112{ }^{\circ} \mathrm{C} .{ }^{1} \mathrm{H}$ NMR $\left(\mathrm{CDCl}_{3}, \delta \mathrm{ppm}\right): 2.324\left(\mathrm{~s}, 6 \mathrm{H}, \mathrm{CH}_{3}\right), 6.894(\mathrm{~d}, 1 \mathrm{H}, \mathrm{Ar}-\mathrm{H})$, $7.1102(\mathrm{~d}, 1 \mathrm{H}, J=16.2 \mathrm{MHz}, \mathrm{CH}=), 7.220-7.547(\mathrm{~m}, 4 \mathrm{H}, \mathrm{Ar}-\mathrm{H}), 8.020(\mathrm{~d}, 1 \mathrm{H}, J=16.1 \mathrm{MHz},=\mathrm{CH}) ;{ }^{13} \mathrm{C}$ NMR ( $\left.\mathrm{CDCl}_{3}, \delta \mathrm{ppm}\right): 20.42\left(2 \mathrm{C}, \mathrm{CH}_{3}\right), 121.20(1 \mathrm{C}, \mathrm{CH}=), 125.57$ (1C), 128.32 (1C), 129.90 (1C), 130.45 (1C), 132.66 (1C), 133.16 (1C), 133.46 (1C), 134.88 (1C), 139.90 (1C), 143.35 (1C), 144.86 $(1 \mathrm{C},=\mathrm{CH}), 152.60(1 \mathrm{C}), 182.44(1 \mathrm{C}, \mathrm{C}=\mathrm{O}) . \mathrm{MS}(\mathrm{EI}) \mathrm{m} / \mathrm{z}: 278.02(32), 276.01(\mathrm{M}+, 100)$; Anal. calcd. for $\mathrm{C}_{15} \mathrm{H}_{13} \mathrm{ClOS}(\%)$ : C, 65.09; H, 4.73. Found: C, 65.03; H, 4.71.

4.3.5 4-(1-(3-Chlorophenyl)-3-(5-chlorothiophen-2-yl)-4,5-dihydro-1H-pyrazol-5-yl)-N,N-

dimethylaniline, 5a: Obtained from 1-(5-chlorothiophen-2-yl)-3-(4-(dimethylamino)phenyl)prop-2-en1-one, 3a (1.49g, $10 \mathrm{mmol})$ and (3-chlorophenyl)hydrazine hydrochloride, $4 \mathbf{b}(1.78 \mathrm{~g}, 10 \mathrm{mmol})$ in 82\% yield, m.p. $103-105{ }^{\circ} \mathrm{C} ;{ }^{1} \mathrm{H}$ NMR $\left(\mathrm{CDCl}_{3}, \delta\right.$ ppm): $3.030\left(\mathrm{~s}, 6 \mathrm{H}, \mathrm{N}-\mathrm{CH}_{3}\right), 3.124(\mathrm{dd}, 1 \mathrm{H}, J=6.6$, $\left.16.0 \mathrm{~Hz}, \mathrm{C}_{4}-\mathrm{H}_{\mathrm{a}}\right), 3.778\left(\mathrm{dd}, 1 \mathrm{H}, J=12.6,7.4 \mathrm{~Hz}, \mathrm{C}_{4}-\mathrm{H}_{\mathrm{b}}\right), 5.247\left(\mathrm{dd}, 1 \mathrm{H}, J=6.3,12.1 \mathrm{~Hz}, \mathrm{C}_{5}-\mathrm{H}\right), 6.715$ (d, 2H, J=7.1 Hz, Ar-H), 6.822 (d, 1H, Ar-H), 6.902 (d, 1H, Ar-H), 7.115 (d, 2H, J=7.2 Hz, Ar-H), 7.235-7.622 (m, 3H, Ar-H); ${ }^{13} \mathrm{C} \mathrm{NMR}\left(\mathrm{CDCl}_{3}, \delta \mathrm{ppm}\right): 40.36\left(2 \mathrm{C}, \mathrm{NCH}_{3}\right), 42.56(1 \mathrm{C}, \mathrm{C}-4), 63.17(1 \mathrm{C}$, C-5), 112.46 (1C), 112.84 (1C), 112.95 (1C), 113.95 (1C), 120.10 (1C), 125.33 (1C), 125.84 (1C), 128.12 (1C), 129.40 (1C), 129.65 (1C), 129.96 (1C), 130.51 (1C), 133.14 (1C), 135.45 (1C), 145.26 (1C), 148.41 (1C), 148.55 (1C, C-3). MS (EI) m/z: 419.05 (11), 417.03 (64), 415.04 (M+, 100); Anal. Calcd. for $\mathrm{C}_{21} \mathrm{H}_{19} \mathrm{Cl}_{2} \mathrm{~N}_{3} \mathrm{~S}(\%)$ : C, 60.58; H, 4.60; N, 10.09; Found: C, 60.51; H, 4.59; N, 10.06 .

4.3.6 3-(5-Chlorothiophen-2-yl)-5-(3,4-dimethoxyphenyl)-1-phenyl-4,5-dihydro-1H-pyrazole, $\quad \mathbf{5 b :}$ Obtained from 1-(5-chlorothiophen-2-yl)-3-(3,4-dimethoxyphenyl)prop-2-en-1-one, 3b (3.08g, 10 mmol) and phenylhydrazine hydrochloride, $4 \mathrm{a}(1.44 \mathrm{~g}, 10 \mathrm{mmole})$ in $86 \%$ yield, m.p. $133-135{ }^{\circ} \mathrm{C} ;{ }^{1} \mathrm{H}$ $\operatorname{NMR}\left(\mathrm{CDCl}_{3}, \delta \mathrm{ppm}\right): 3.113\left(\mathrm{dd}, 1 \mathrm{H}, J=6.5,16.3 \mathrm{~Hz}, \mathrm{C}_{4}-\mathrm{H}_{\mathrm{a}}\right), 3.769\left(\mathrm{dd}, 1 \mathrm{H}, J=12.1,7.0 \mathrm{~Hz}, \mathrm{C}_{4}-\mathrm{H}_{\mathrm{b}}\right)$, $3.850\left(\mathrm{~s}, 6 \mathrm{H}, \mathrm{OCH}_{3}\right), 5.238\left(\mathrm{dd}, 1 \mathrm{H}, J=6.1,12.3 \mathrm{~Hz}, \mathrm{C}_{5}-\mathrm{H}\right), 6.786-7.082(\mathrm{~m}, 8 \mathrm{H}, \mathrm{Ar}-\mathrm{H}), 7.120-7.196$ (m, 2H, Ar-H); ${ }^{13} \mathrm{C} \mathrm{NMR}\left(\mathrm{CDCl}_{3}, \delta \mathrm{ppm}\right): 42.60(1 \mathrm{C}, \mathrm{C}-4), 55.45\left(2 \mathrm{C}, \mathrm{OCH}_{3}\right), 63.25(1 \mathrm{C}, \mathrm{C}-5), 108.90$ (1C), 115.26 (1C), 115.54 (1C), 119.95 (1C), 120.14 (1C), 125.46 (1C), 125.73 (1C), 128.19 (1C), 128.60 (1C), 129.52 (1C), 129.70 (1C), 130.66 (1C), 134.17 (1C), 142.73 (1C), 143.82 (1C), 148.55 (1C), 148.93 (1C, C-3). MS (EI) m/z: 400.05 (34), 398.07 (M+, 100); Anal. Calcd. for $\mathrm{C}_{21} \mathrm{H}_{19} \mathrm{ClN}_{2} \mathrm{O}_{2} \mathrm{~S}$ (\%): C, 63.23; H, 4.80; N, 7.02; Found: C, 63.18; H, 4.79; N, 7.00.

\subsubsection{1-(3-Chlorophenyl)-3-(5-chlorothiophen-2-yl)-5-(3,4-dimethoxyphenyl)-4,5-dihydro-1H-}

pyrazole, 5c: Obtained from 1-(5-chlorothiophen-2-yl)-3-(3,4-dimethoxyphenyl)prop-2-en-1-one, 3b $(3.08 \mathrm{~g}, 10 \mathrm{mmol})$ and (3-chlorophenyl)hydrazine hydrochloride, $4 \mathbf{b}(1.78 \mathrm{~g}, 10 \mathrm{mmol})$ in $80 \%$ yield, m.p. $144-146{ }^{\circ} \mathrm{C} ;{ }^{1} \mathrm{H}$ NMR $\left(\mathrm{CDCl}_{3}, \delta\right.$ ppm): 3.117 (dd, $\left.1 \mathrm{H}, J=6.1,16.0 \mathrm{~Hz}, \mathrm{C}_{4}-\mathrm{H}_{\mathrm{a}}\right), 3.764(\mathrm{dd}, 1 \mathrm{H}$, $\left.J=12.0,7.3 \mathrm{~Hz}, \mathrm{C}_{4}-\mathrm{H}_{\mathrm{b}}\right), 3.845\left(\mathrm{~s}, 6 \mathrm{H}, \mathrm{OCH}_{3}\right), 5.241\left(\mathrm{dd}, 1 \mathrm{H}, J=6.2,12.4 \mathrm{~Hz}, \mathrm{C}_{5}-\mathrm{H}\right), 6.806-6.992(\mathrm{~m}$, $5 \mathrm{H}, \mathrm{Ar}-\mathrm{H}), 7.231-7.645(\mathrm{~m}, 4 \mathrm{H}, \mathrm{Ar}-\mathrm{H}) ;{ }^{13} \mathrm{C} \mathrm{NMR}\left(\mathrm{CDCl}_{3}, \delta \mathrm{ppm}\right): 42.64(1 \mathrm{C}, \mathrm{C}-4), 55.48\left(2 \mathrm{C}, \mathrm{OCH}_{3}\right)$, 63.32 (1C, C-5), 109.14 (1C), 112.23 (1C), 114.67 (1C), 118.83 (1C), 120.97 (1C), 125.51 (1C), 125.82 (1C), 128.10 (1C), 128.87 (1C), 130.10 (1C), 130.22 (1C), 134.77 (1C), 136.54 (1C), 144.70 (1C), 147.80 (1C), 148.75 (1C, C-3), 149.60 (1C). MS (EI) m/z: 436.02 (11), 434.03 (64), 432.01 (M+, 100); Anal. Calcd. for $\mathrm{C}_{21} \mathrm{H}_{18} \mathrm{Cl}_{2} \mathrm{~N}_{2} \mathrm{O}_{2} \mathrm{~S}(\%)$ : C, 58.21; H, 4.19; N, 6.46; Found: C, 58.16; H, 4.18; N, 6.44.

4.3.8 5-(4-Bromophenyl)-3-(5-chlorothiophen-2-yl)-1-phenyl-4,5-dihydro-1H-pyrazole,

5d: Obtained from 3-(4-bromophenyl)-1-(5-chlorothiophen-2-yl)prop-2-en-1-one, 3c (3.25g, $10 \mathrm{mmol})$ and phenylhydrazine hydrochloride, 4a $(1.44 \mathrm{~g}, 10$ mmole $)$ in $72 \%$ yield, m.p. $177-170{ }^{\circ} \mathrm{C} ;{ }^{1} \mathrm{H}$ NMR $\left(\mathrm{CDCl}_{3}, \delta \mathrm{ppm}\right): 3.128\left(\mathrm{dd}, 1 \mathrm{H}, J=6.3,16.7 \mathrm{~Hz}, \mathrm{C}_{4}-\mathrm{H}_{\mathrm{a}}\right), 3.765\left(\mathrm{dd}, 1 \mathrm{H}, J=12.2,7.1 \mathrm{~Hz}, \mathrm{C}_{4}-\mathrm{H}_{\mathrm{b}}\right), 5.251$ (dd, 1H, $\left.J=6.0,12.3 \mathrm{~Hz}, \mathrm{C}_{5}-\mathrm{H}\right), 6.902-6.984(\mathrm{~m}, 5 \mathrm{H}, \mathrm{Ar}-\mathrm{H}), 7.114(\mathrm{~d}, 2 \mathrm{H}, J=7.2 \mathrm{~Hz}, \mathrm{Ar}-\mathrm{H}), 7.222-$ $7.384(\mathrm{~m}, 2 \mathrm{H}, \mathrm{Ar}-\mathrm{H}), 7.841$ (d, 2H, $J=7.1 \mathrm{~Hz}, \mathrm{Ar}-\mathrm{H}) ;{ }^{13} \mathrm{C} \mathrm{NMR}\left(\mathrm{CDCl}_{3}, \delta \mathrm{ppm}\right): 42.54$ (1C, C-4), 63.30 (1C, C-5), 116.42 (1C), 116.80 (1C), 120.75 (1C), 121.76 (1C), 125.25 (1C), 125.76 (1C), 127.30 (1C), 127.55 (1C), 128.24 (1C), 129.18 (1C), 129.70 (1C), 130.63 (1C), 131.40 (1C), 131.52 (1C), 142.90 (1C), 143.21 (1C), 148.85 (1C, C-3). MS (EI) m/z: 419.97 (31), 417.96 (98), 415.98 (M+, 100); Anal. Calcd. for $\mathrm{C}_{19} \mathrm{H}_{14} \mathrm{BrClN}_{2} \mathrm{~S}(\%)$ : C, 54.63; H, 3.38; N, 6.71; Found: C, 54.59; H, 3.37; N, 6.69. 
4.3.9 5-(4-Bromophenyl)-1-(3-chlorophenyl)-3-(5-chlorothiophen-2-yl)-4,5-dihydro-1H-pyrazole, 5e: Obtained from 3-(4-bromophenyl)-1-(5-chlorothiophen-2-yl)prop-2-en-1-one, 3c (3.25g, $10 \mathrm{mmol})$ and (3-chlorophenyl)hydrazine hydrochloride, $4 \mathbf{b}(1.78 \mathrm{~g}, 10 \mathrm{mmol})$ in $76 \%$ yield, m.p. $135-137{ }^{\circ} \mathrm{C}$; ${ }^{1} \mathrm{H}$ NMR $\left(\mathrm{CDCl}_{3}, \delta \mathrm{ppm}\right): 3.121\left(\mathrm{dd}, 1 \mathrm{H}, J=6.3,16.1 \mathrm{~Hz}, \mathrm{C}_{4}-\mathrm{H}_{\mathrm{a}}\right), 3.770\left(\mathrm{dd}, 1 \mathrm{H}, J=12.2,7.5 \mathrm{~Hz}, \mathrm{C}_{4}{ }^{-}\right.$ $\left.\mathrm{H}_{\mathrm{b}}\right), 5.242\left(\mathrm{dd}, 1 \mathrm{H}, J=6.1,12.4 \mathrm{~Hz}, \mathrm{C}_{5}-\mathrm{H}\right), 6.830-6.845$ (m, 2H, Ar-H), $7.112(\mathrm{~d}, 2 \mathrm{H}, J=7.2 \mathrm{~Hz}, \mathrm{Ar}-\mathrm{H})$, 7.302-7.664 (m, 4H, Ar-H), $7.846(\mathrm{~d}, 2 \mathrm{H}, J=7.1 \mathrm{~Hz}, \mathrm{Ar}-\mathrm{H}) ;{ }^{13} \mathrm{C} \mathrm{NMR}\left(\mathrm{CDCl}_{3}, \delta \mathrm{ppm}\right): 42.71(1 \mathrm{C}, \mathrm{C}-$ 4), 63.30 (1C, C-5), 112.51 (1C), 113.88 (1C), 120.27 (1C), 120.66 (1C), 121.24 (1C), 125.30 (1C), 125.55 (1C), 128.16 (1C), 127.61 (1C), 127.93 (1C), 130.34 (1C), 131.18 (1C), 131.42 (1C), 136.40 (1C), 142.40 (1C), 145.21 (1C), 148.60 (1C, C-3). MS (EI) $\mathrm{m} / z: 453.93$ (62), 451.93 (97), 451.93 (63), $449.95(\mathrm{M}+, 100)$; Anal. Calcd. for $\mathrm{C}_{19} \mathrm{H}_{13} \mathrm{BrCl}_{2} \mathrm{~N}_{2} \mathrm{~S}(\%)$ : C, 50.47; H, 2.90; N, 6.20; Found: C, 50.42; $\mathrm{H}, 2.90 ; \mathrm{N}, 6.18$.

4.3.10 3-(5-Chlorothiophen-2-yl)-5-(2,4-dimethylphenyl)-1-phenyl-4,5-dihydro-1H-pyrazole, $\quad$ 5f: Obtained from 1-(5-chlorothiophen-2-yl)-3-(2,4-dimethylphenyl)prop-2-en-1-one, 3d (2.76g, 10 $\mathrm{mmol})$ and phenylhydrazine hydrochloride, $4 \mathrm{a}(1.44 \mathrm{~g}, 10 \mathrm{mmole})$ in $89 \%$ yield, m.p. $168-169{ }^{\circ} \mathrm{C} ;{ }^{1} \mathrm{H}$ NMR $\left(\mathrm{CDCl}_{3}, \delta \mathrm{ppm}\right): 2.295\left(\mathrm{~s}, 6 \mathrm{H}, \mathrm{CH}_{3}\right), 3.122\left(\mathrm{dd}, 1 \mathrm{H}, J=7.2,16.8 \mathrm{~Hz}, \mathrm{C}_{4}-\mathrm{H}_{\mathrm{a}}\right), 3.740(\mathrm{dd}, 1 \mathrm{H}$, $\left.J=12.0,7.1 \mathrm{~Hz}, \mathrm{C}_{4}-\mathrm{H}_{\mathrm{b}}\right), 5.240\left(\mathrm{dd}, 1 \mathrm{H}, J=6.4,12.0 \mathrm{~Hz}, \mathrm{C}_{5}-\mathrm{H}\right), 6.781-6.982(\mathrm{~m}, 6 \mathrm{H}, \mathrm{Ar}-\mathrm{H}), 7.102-7.228$

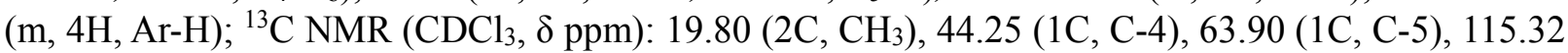
(1C), 115.46 (1C), 120.22 (1C), 125.03 (1C), 125.28 (1C), 125.59 (1C), 126.48 (1C), 128.50 (1C), 129.16 (1C), 129.27 (1C), 130.55 (1C), 130.95 (1C), 134.24 (1C), 135.66 (1C), 138.41 (1C), 142.98 (1C), 147.30 (1C, C-3). MS (EI) m/z: 368.09 (32), 366.07 (M+, 100); Anal. Calcd. for $\mathrm{C}_{21} \mathrm{H}_{19} \mathrm{ClN}_{2} \mathrm{~S}$ (\%): C, 68.75; H, 5.22; N, 7.64; Found: C, 68.69; H, 5.20; N, 7.62.

4.3.11 1-(3-Chlorophenyl)-3-(5-chlorothiophen-2-yl)-5-(2,4-dimethylphenyl)-4,5-dihydro-1Hpyrazole, 5g: Obtained from 1-(5-chlorothiophen-2-yl)-3-(2,4-dimethylphenyl)prop-2-en-1-one, 3d $(2.76 \mathrm{~g}, 10 \mathrm{mmol})$ and (3-chlorophenyl)hydrazine hydrochloride, $4 \mathbf{b}(1.78 \mathrm{~g}, 10 \mathrm{mmol})$ in $77 \%$ yield, m.p. $142-144{ }^{\circ} \mathrm{C}$; ${ }^{1} \mathrm{H}$ NMR $\left(\mathrm{CDCl}_{3}, \delta \mathrm{ppm}\right): 2.230\left(\mathrm{~s}, 6 \mathrm{H}, \mathrm{CH}_{3}\right), 3.120\left(\mathrm{dd}, 1 \mathrm{H}, J=6.9,16.1 \mathrm{~Hz}, \mathrm{C}_{4}-\right.$ $\left.\mathrm{H}_{\mathrm{a}}\right), 3.780\left(\mathrm{dd}, 1 \mathrm{H}, J=12.5,7.0 \mathrm{~Hz}, \mathrm{C}_{4}-\mathrm{H}_{\mathrm{b}}\right), 5.242\left(\mathrm{dd}, 1 \mathrm{H}, J=6.1,12.2 \mathrm{~Hz}, \mathrm{C}_{5}-\mathrm{H}\right), 6.785-6.816(\mathrm{~m}, 3 \mathrm{H}$,

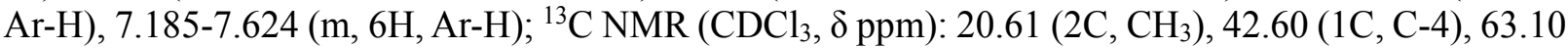
(1C, C-5), 112.35 (1C), 113.45 (1C), 120.15 (1C), 125.20 (1C), 125.61 (1C), 126.04 (1C), 126.53 (1C), 127.19 (1C), 129.70 (1C), 129.97 (1C), 130.52 (1C), 134.15 (1C), 135.12 (1C), 136.60 (1C), 138.41 (1C), 144.80 (1C), 149.64 (1C, C-3). MS (EI) m/z: 404.04 (10), 402.03 (34), 400.04 (M+, 100); Anal. Calcd. for $\mathrm{C}_{21} \mathrm{H}_{18} \mathrm{Cl}_{2} \mathrm{~N}_{2} \mathrm{~S}(\%)$ : C, 62.85; H, 4.52; N, 6.98; Found: C, 62.80; H, 4.51; N, 6.96.

\section{References}

1 Darja G., Lucija P.M., Marija S.D. (2014) Bioactivation potential of thiophene-containing drugs. Chem. Res. Toxicol. 27 (8) 1344-1358.

2 Manjunath B.C., Manjula M., Raghavendra K.R., Ajay Kumar K., Lokanath N.K. (2014) 4-(Thiophen2-yl)-2-[4-(trifluoromethyl)-phenyl]-2,3-dihydro-1,5-benzothiazepine. Acta Cryst. Sect. E, 70 (Part 3) o261-o261.

3 Kumar G.V., Govindaraju M., Renuka N., Khatoon B.B.A., Mylarappa B.N., Kumar K.A. (2012) Synthesis of 1,3,5-triaryl-4,6-dioxo-pyrrolo[3,4- $d]$-7,8-dihydropyrzoles and their antimicrobial and antioxidant activity. Rasayan J. Chem. 5 (3) 338-342.

4 Ajay Kumar K., Govindaraju M., Vasantha Kumar G. (2010) Synthesis of isoxazoles via 1,3-dipolar cycloaddition reactions and their antimicrobial activity. Ind. J. Heterocycl. Chem. 20 (4) 183-184.

5 Ajay Kumar K., Lokanatha Rai K.M., Vasanth Kumar G., Mylarappa B.N. (2012) A facile route for the synthesis of ethyl $\mathrm{N}$-aryl-2,6-dioxo-piperid-3-ene-4-carboxylates and their biological activity. Int. J. Pharm. Pharm. Sci. 4 (Suppl 4) 564-568.

6 Ajay Kumar K., Lokanatha Rai K.M. (2004) Synthesis and evaluation of antimicrobial activity of 4,5dihydro-12,4-oxadiazoles, Bulg. Chem. Commun. 36 (4) 249-252.

7 Naveen S., Dileep Kumar A., Ajay Kumar K., Manjunath H.R., Lokanath N. K., Warad I. (2016) (E)3-(2,3-Dichlorophenyl)-1-(4-fluorophenyl)prop-2-en-1-one. IUCrData, 1 (11), x161800- x161800. 
8 Bukhari S.N.A., Jasamai M., Jantan I. (2012) Synthesis and biological evaluation of chalcone derivatives (mini review). Mini-Rev.Med. Chem. 12 (3) 1394-1403.

9 Kumar V., Kaur K., Gupta G.K., Sharma A.K. (2013) Pyrazole containing natural products: synthetic preview and biological significance. Eur. J. Med. Chem. 69 735-753.

10 Prabhashankar J., Govindappa V.K., Kariyappa A.K. (2013) Synthesis of 3,4-diaryl-1-phenyl-4,5dihydro-1H-pyrazole-5-carbonitriles via 1,3-dipolar cycloaddition reactions, Turk. J. Chem. 37 (5) 853-857.

11 Al-Saleh F.S., Al Khawaja I.K., Joule J.A. (1981) Synthesis of 4-acyl- and 4-alkoxycarbonylpyrazoles, J. Chem. Soc. Perkin Trans. 1 642-645.

12 Govindaraju M., Mylarappa B.N., Ajay Kumar K. (2013) Synthesis of novel pyrazole derivatives and their efficacy as antimicrobial agents. Int. J. Pharm. Pharm. Sci. 5 (4), 734-737.

13 Nagamallu R., Srinivasan B., Ningappa M.B., Kariyappa A.K. (2016) Synthesis of novel coumarin appended bis(formylpyrazole) derivatives: Studies on their antimicrobial and oxidant activities, Bioorg. Med. Chem. Lett. 26 (2) 690-694.

14 Stauffer S.R., Coletta C.J., Tedesco R., Nishiguchi G., Carlson K., Sun J., Katzenellenbogen B.S., Katzenellenbogen J.A. (2000) Pyrazole ligands: structure-affinity/activity relationships and estrogen receptor-alpha-selective agonists. J. Med. Chem. 43 (26) 4934-4934.

15 Vijaychand S.P., Pavithra G., Raghavendra K.R., Ajay Kumar K. (2015) An efficient route to synthesis of pyrazoline carboxamides bearing thiophene moiety as antimicrobial agents. Der Pharma Chem. 7 (4) 85-89.

16 Christina Z., Florea D., Constantin D., Mircea I., Maria M., Isabela T., George M.N. (2014) Synthesis and biological screening of some 2-(1H-pyrazol-1-yl)-acetamides as lidocaine analogue. Ind. J. Chem. 53B (6) 733-739.

17 Pavithra G., Kariyappa A.K. (2016) Synthesis of bioactive furan derivatised pyrazole carboxamides and their antimicrobial and antioxidant activities, Der Pharma Chem. 8 (8) 6-11.

18 Katoch-Rouse R., Pavlova O.A., Caulder T., Hoffmann A.F., Mukhin A.G., Horti A.G. (2003) Synthesis, structure-activity relationship, and evaluation of SR141716 analogues: Development of central cannabinoid receptor ligands with lower lipophilicity. J. Med. Chem. 46 (4) 642-645.

19 Raghavendra K.R., Renuka N., Ajay Kumar K., Shashikanth S. (2017) An accessible route for the synthesis of novel lignan derivatives and their biological evaluation. Pharm. Chem. J. 51 (8) 661-669.

20 Raghavendra K.R., Renuka N., Kameshwar V. H., Srinivasan B., Ajay Kumar K., Shashikanth S. (2016) Synthesis of lignan conjugate via cyclopropanation: Antimicrobial and antioxidant studies, Bioorg. Med. Chem. Lett. 26 (15) 3621-3625.

21 Mahadevaswamy L.D., Kariyappa A.K. (2017) An environmentally benign lemon juice mediated synthesis of novel furan conjugated pyrazole derivatives and their biological evaluation. Pharm. Chem. J. 51 (8) 670-677.

22 Lokeshwari D.M., Kameshwar V.H., Kariyappa A.K, (2017) Synthesis of furan tethered 2-pyrazolines via 1,3-dipolar cycloaddition reactions: In vitro evaluation for their antioxidant and antimicrobial activities, molecular docking and ADMET studies, Biointerface Res. App. Chem. 7(5) 2158-2165.

23 Prabhudeva M.G., Bharath S., Kumar A.D., Naveen S., Lokanath N.K., Mylarappa B.N., Kumar K.A. (2017) Design and environmentally benign synthesis of novel thiophene appended pyrazole derivatives as anti-inflammatory and radical scavenging agents: crystallographic, in silico modeling, docking and SAR characterization. Bioorg. Chem. 73 109-120.

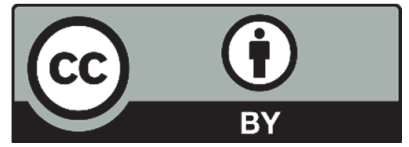

(C) 2018 by the authors; licensee Growing Science, Canada. This is an open access article distributed under the terms and conditions of the Creative Commons Attribution (CC-BY) license (http://creativecommons.org/licenses/by/4.0/). 